\title{
THE LEFSCHETZ PROPERTY FOR FAMILIES OF CURVES
}

\author{
JÁNOS KOLLÁR
}

By the Lefschetz hyperplane theorem, if $X \subset \mathbb{P}^{N}$ is a smooth, projective variety and $C:=X \cap L$ is a positive dimensional intersection of $X$ with a linear subspace, then the natural map

$$
\pi_{1}(C) \rightarrow \pi_{1}(X) \text { is surjective. }
$$

The same conclusion holds if $X$ is quasi projective, but here $C$ has to be an intersection of $X$ with a linear subspace in general position. The aim of this note is to study families of curves $\left\{C_{m}: m \in M\right\}$ that satisfy this Lefschetz-type property. Such results were proved in the papers [Kol00, Kol03]. Arithmetic applications are given in Kol00, CT00, KS03 and Kol13 studies this question for homogeneous spaces. Related results are in [BdS09, BK08, BK12].

Definition 1. A family of schemes over a normal variety $X$ over $\mathbb{C}$ is a diagram

$$
M \stackrel{p}{\leftarrow} C_{M} \stackrel{u}{\rightarrow} X .
$$

Our main interest is in families where $M$ is irreducible and $p$ is flat with irreducible fibers. The family (11) satisfies the Lefschetz property if the following holds.

For every Zariski open subset $\emptyset \neq X^{0} \subset X$ there is a Zariski open subset $\emptyset \neq M^{0} \subset M$ such that, for every $m \in M^{0}$, the induced map

$$
u\left(X^{0}, m\right)_{*}: \pi_{1}\left(C_{m} \cap u^{-1}\left(X^{0}\right)\right) \rightarrow \pi_{1}\left(X^{0}\right) \text { is surjective. }
$$

We say that (1.1) satisfies the weak Lefschetz property if there is a constant $N$ (independent of $\left.X^{0}\right)$ such that, for suitable choice of $M^{0}$, the image of $u\left(X^{0}, m\right)_{*}$ has index at most $N$ in $\pi_{1}\left(X^{0}\right)$.

Notes. We ignore the base point since the surjectivity of the maps between the fundamental groups of connected schemes does not depend on the choice of a base point. The Lefschetz properties over arbitrary base fields are considered in (21).

Our main Theorem 5 is somewhat technical, though I believe it to be essentially optimal. The original arguments of [Kol00, Kol03] need high degree very free rational curves. By contrast, the current proof frequently works for the lowest degree free curves. As an illustration, a simple yet nontrivial example is given by lines on hypersurfaces.

Corollary 2. Let $X \subset \mathbb{P}^{n}$ be a smooth hypersurface of degree $d$ over $\mathbb{C}$. The following are equivalent.

(1) The family of lines has the Lefschetz property.

(2) The family of lines has the weak Lefschetz property.

(3) $d \leq n-2$ or $X$ is a line in $\mathbb{P}^{2}$.

Let us start with some situations when the Lefschetz property fails. 
Example 3. Let $M \leftarrow C_{M} \stackrel{u}{\rightarrow} X$ be a flat, irreducible family of irreducible varieties.

(3.1) Assume that $u$ is not dominant. Then any $X^{0} \subset X \backslash \overline{u\left(C_{M}\right)}$ with infinite fundamental group shows that the weak Lefschetz property does not hold.

(3.2) Assume that there is an open subset $X^{*}$ and a dominant morphism to a positive dimensional variety $q: X^{*} \rightarrow Z^{*}$ such that every $X^{*} \cap C_{m}$ is contained in a fiber of $q$ for general $m \in M$. (We say that $X$ is generically $C_{M}$-connected if there is no such map $q: X^{*} \rightarrow Z^{*}$; see (12) for a better definition.)

Let $Z^{0} \subset Z^{*}$ be an open subset and $X^{0}:=q^{-1}\left(Z^{0}\right)$. Then

$$
\operatorname{im}\left[\pi_{1}\left(C_{m} \cap u^{-1}\left(X^{0}\right)\right) \rightarrow \pi_{1}\left(X^{0}\right)\right] \subset \operatorname{ker}\left[\pi_{1}\left(X^{0}\right) \rightarrow \pi_{1}\left(Z^{0}\right)\right] .
$$

If $Z^{0}$ has infinite fundamental group then the weak Lefschetz property fails.

(3.3) Assume that $u: C_{M} \rightarrow X$ does not have geometrically irreducible generic fiber. Then there is a nontrivial Stein factorization $u: C_{m} \stackrel{w}{\rightarrow} Y \stackrel{v}{\rightarrow} X$ where $v$ is finite, generically étale of degree $>1$ and $w$ has geometrically irreducible generic fiber (see [Kol03, Lem.9] for the non-proper variant used here). Let $X^{0} \subset X$ be an open set such that $v$ is étale over $X^{0}$ and $Y^{0}:=v^{-1}\left(X^{0}\right)$. For general $m \in M$, the induced map $C_{m} \rightarrow X$ factors through $Y$, hence

$$
\operatorname{im}\left[\pi_{1}\left(C_{m} \cap u^{-1}\left(X^{0}\right)\right) \rightarrow \pi_{1}\left(X^{0}\right)\right] \subset \operatorname{im}\left[\pi_{1}\left(Y^{0}\right) \rightarrow \pi_{1}\left(X^{0}\right)\right] \subsetneq \pi_{1}\left(X^{0}\right) .
$$

In this case the Lefschetz property fails but the weak variant could hold with $N=$ the number of geometric irreducible components of the generic fiber of $u$. More generally, we see that the weak Lefschetz property for $M \leftarrow C_{M} \stackrel{u}{\rightarrow} X$ is equivalent to the weak Lefschetz property for $M \leftarrow C_{M} \stackrel{w}{\rightarrow} Y$. The advantage is that $w: C_{M} \rightarrow Y$ has geometrically irreducible generic fiber.

(3.4) An extreme case of the above is when $u: C_{M} \rightarrow X$ is generically finite. Then $w: C_{M} \rightarrow Y$ is birational and (32) applies to $p: C_{M} \rightarrow M$. Thus the weak Lefschetz property does not hold for $M \leftarrow C_{M} \stackrel{u}{\rightarrow} X$. (A trivial exception is when $M$ is a single point, giving the case $X=\mathbb{P}^{1}$ in (2)3).)

(3.5) A difficulty in using the reduction method of (3.3) is that being generically $C_{M}$-connected changes as we pass from $X$ to $Y$. A typical example is the following.

For some $n \geq 2$ set $X=S^{2} \mathbb{P}^{n} \backslash$ (diagonal). Its universal cover is $\tilde{X}=\mathbb{P}^{n} \times$ $\mathbb{P}^{n} \backslash$ (diagonal). Let $\tilde{u}: \tilde{C}_{L} \rightarrow \tilde{X}$ be the family of lines that are contained in some $\mathbb{P}^{n} \times\{$ point $\}$ and $u: C_{L} \rightarrow X$ the corresponding family of lines in $X$. Note that $X$ is generically $C_{L}$-connected but $\tilde{X}$ is not generically $\tilde{C}_{L}$-connected.

Since each point in $X$ has 2 preimages in $\tilde{X}$, each fiber of $u$ has 2 irreducible components.

For an open set $W \subset \mathbb{P}^{n}$ let $X_{W} \subset X$ denote the image of $W \times W$. Then there is an extension

$$
1 \rightarrow \pi_{1}(W)+\pi_{1}(W) \rightarrow \pi_{1}\left(X_{W}\right) \rightarrow\{ \pm 1\} \rightarrow 1
$$

and for any line $C_{m}$, the image of $\pi_{1}\left(C_{m} \cap X_{W}\right)$ lies in the first summand $\pi_{1}(W)$. Thus if $\pi_{1}(W)$ is infinite then even the weak Lefschetz property fails.

(3.6) Continuing with the previous example, let $\tilde{w}: \tilde{C}_{Q} \rightarrow \tilde{X}$ be the family of conics (that is rational curves of bidegree $(1,1)$ ) and $w: C_{Q} \rightarrow X$ the corresponding family of conics in $X$. Here both $\tilde{w}$ and $w$ have connected fibers. It follows from our results that $\tilde{w}: \tilde{C}_{Q} \rightarrow \tilde{X}$ satisfies the Lefschetz property but $w: C_{Q} \rightarrow X$ only satisfies the weak Lefschetz property (with $N=2$ ). 
(3.7) The Lefschetz properties are really about small open subsets of $X$ and of $C_{M}$. To see this, let $u: Y \rightarrow X$ be a morphism between normal varieties, $X^{0} \subset X$ an open subvariety and $Y^{0}:=u^{-1}\left(X^{0}\right)$. Then $\pi_{1}\left(X^{0}\right) \rightarrow \pi_{1}(X)$ is surjective (cf. [Kol95, 2.10]), thus the index in Definition 11 increases as we pass from $X$ to $X^{0}$. That is,

$$
\left|\pi_{1}\left(X^{0}\right): \operatorname{im}\left[\pi_{1}\left(Y^{0}\right) \rightarrow \pi_{1}\left(X^{0}\right)\right]\right| \geq\left|\pi_{1}(X): \operatorname{im}\left[\pi_{1}(Y) \rightarrow \pi_{1}(X)\right]\right| .
$$

Next let $C_{M}^{0} \subset C_{M}$ be a dense open subset. Then $C_{m}^{0}$ is a dense open subset of $C_{m}$ for general $m \in M$. Thus, if $C_{m}$ is normal, then $\pi_{1}\left(C_{m}^{0}\right) \rightarrow \pi_{1}\left(C_{m}\right)$ is surjective by [Kol95, 2.10], so

$$
\operatorname{im}\left[\pi_{1}\left(C_{m}^{0}\right) \rightarrow \pi_{1}\left(X^{0}\right)\right]=\operatorname{im}\left[\pi_{1}\left(C_{m}\right) \rightarrow \pi_{1}\left(X^{0}\right)\right] .
$$

Our first result says that these examples almost explain every failure of the Lefschetz property.

Proposition 4. Let $X$ be a normal variety over $\mathbb{C}$ and $M \leftarrow C_{M} \rightarrow X$ a flat, irreducible family of irreducible varieties. Then each of the following statements implies the next.

(1) $M \leftarrow C_{M} \rightarrow X$ satisfies the Lefschetz property.

(2) $C_{M} \rightarrow X$ is dominant, has geometrically irreducible generic fiber and $X$ is generically $C_{M}$-connected.

(3) $M \leftarrow C_{M} \rightarrow X$ satisfies the weak Lefschetz property.

In any concrete situation is usually easy to check that $C_{M} \rightarrow X$ is dominant and has geometrically irreducible generic fiber. Being generically $C_{M}$-connected is not always clear but it holds if $X$ is smooth, proper, has Picard number 1 and $M \leftarrow C_{M} \stackrel{u}{\rightarrow} X$ is a locally complete family of free curves; see [Kol96, IV.4.14].

Sometimes the difference between the Lefschetz property and the weak Lefschetz property is minor, but in the arithmetic applications [Kol00, CT00, KS03, having surjectivity is essential. The following main technical result says that if we avoid the bad situations (3,1-3) and we have surjectivity for $\pi_{1}(X)$ itself then the Lefschetz property holds. More generally, the extent of any failure of the Lefschetz property is determined by $X$ itself.

Theorem 5. Let $M \stackrel{p}{\leftarrow} C_{M} \stackrel{u}{\rightarrow} X$ be a family of varieties over a smooth (not necessarily proper) variety $X$, defined over $\mathbb{C}$. Assume that

(1) $p$ and $u$ are both smooth with irreducible fibers,

(2) $u$ is surjective and

(3) $X$ is generically $C_{M}$-connected.

Let $j: X^{0} \hookrightarrow X$ be an open subset and $j_{*}: \pi_{1}\left(X^{0}\right) \rightarrow \pi_{1}(X)$ the induced map on the fundamental groups. Then there is an open subset $\emptyset \neq M^{0} \subset M$ such that

$$
\operatorname{im}\left[\pi_{1}\left(C_{m} \cap u^{-1}\left(X^{0}\right)\right) \rightarrow \pi_{1}\left(X^{0}\right)\right]=j_{*}^{-1}\left(\operatorname{im}\left[\pi_{1}\left(C_{m}\right) \rightarrow \pi_{1}(X)\right]\right)
$$

for every $m \in M^{0}$.

We already know from Proposition 4 that both images in (5.4) are finite index subgroups. Thus (5) is equivalent to the equality

$$
\left|\pi_{1}\left(X^{0}\right): \operatorname{im}\left[\pi_{1}\left(C_{m} \cap u^{-1}\left(X^{0}\right)\right) \rightarrow \pi_{1}\left(X^{0}\right)\right]\right|=\left|\pi_{1}(X): \operatorname{im}\left[\pi_{1}\left(C_{m}\right) \rightarrow \pi_{1}(X)\right]\right| .
$$


If $X$ is simply connected then the right hand side of (5.4) equals $\pi_{1}\left(X^{0}\right)$. Thus, in this case, we assert that $\pi_{1}\left(C_{m} \cap u^{-1}\left(X^{0}\right)\right) \rightarrow \pi_{1}\left(X^{0}\right)$ is onto for every $m \in M^{0}$. The latter is exactly the Lefschetz property.

When applying Theorem 5 to any family $M \stackrel{p}{\leftarrow} C_{M} \stackrel{u}{\rightarrow} X$, we first replace $M$ by $M \backslash$ Sing $M$, then replace $C_{M}$ by the largest open subset $C_{M}^{0}$ where $p$ and $u$ are both smooth and finally replace $X$ by $u\left(C_{M}^{0}\right)$. The first step is entirely harmless. The key question is to understand how large $X \backslash u\left(C_{M}^{0}\right)$ is; only the divisors contained in it matter.

As a significant example, let $X$ be smooth, proper and $M \subset \operatorname{Mor}\left(\mathbb{P}^{1}, X\right)$ a nonempty, irreducible, open subset with universal morphism $u: M \times \mathbb{P}^{1} \rightarrow X$. For $x \in X$ let $M_{x} \subset M$ be the set of maps $[f] \in M$ such that $f(0: 1)=x$.

Corollary 6. Let $X$ be a normal, proper variety and $M \subset \operatorname{Mor}\left(\mathbb{P}^{1}, X\right)$ a nonempty, irreducible, open subset parametrizing free maps with universal morphism $u: M \times$ $\mathbb{P}^{1} \rightarrow X$. Assume that

(1) $X \backslash \operatorname{Sing} X$ is simply connected,

(2) $X \backslash u\left(M \times \mathbb{P}^{1}\right)$ has codimension $\geq 2$ and

(3) $X$ is generically $M \times \mathbb{P}^{1}$-connected.

Then $u: M \times \mathbb{P}^{1} \rightarrow X$ satisfies the Lefschetz property iff $M_{x}$ is irreducible for general $x \in X$.

Proof. The projection $M \times \mathbb{P}^{1} \rightarrow M$ is obviously smooth and $u$ is smooth by Kol96, I.3.5.4] since we parametrize free morphism. We apply Theorem 5 to $X^{*}:=$ $u\left(M \times \mathbb{P}^{1}\right)$ replacing $X$. By assumption, $X^{*}$ is obtained from the simply connected smooth variety $X \backslash \operatorname{Sing} X$ by removing a closed subscheme of codimension $\geq 2$. Thus $X^{*}$ is also simply connected and hence the right hand side of $(5.3)$ equals $\pi_{1}\left(X^{0}\right)$.

Remark 7. If $M_{x}$ is reducible for general $x \in X$ then instead of $u: M \times \mathbb{P}^{1} \rightarrow X$ one can work with the family of rational curves obtained by smoothing a bouquet of rational curves through $x$, one from each irreducible component of $M_{x}$.

Note that the assumptions (6, $1-3)$ hold if $X$ is smooth and has Picard number $\rho(X)=1$. Thus we get the following.

Corollary 8. Let $X$ be a smooth proper variety with $\rho(X)=1$. Let $M \subset$ $\operatorname{Mor}\left(\mathbb{P}^{1}, X\right)$ be a nonempty, irreducible, open subset parametrizing free maps. Then the universal morphism $u: M \times \mathbb{P}^{1} \rightarrow X$ satisfies the Lefschetz property iff $M_{x}$ is irreducible for general $x \in X$.

9 (Proof of Corollary 2). Let $M \leftarrow C_{M} \stackrel{u}{\rightarrow} X$ be the universal family of lines. Let $x \in X$ be a point. After a coordinate change, we may assume that $x=(1: 0: \cdots: 0)$. Write the equation of $X_{d}$ as

$$
g_{1}\left(x_{1}, \ldots, x_{n}\right) x_{0}^{d-1}+\cdots+g_{d}\left(x_{1}, \ldots, x_{n}\right) .
$$

The family of lines in $X$ through $x$ is then given by the equations

$$
M_{x}:=\left(g_{1}=\cdots=g_{d}\right) \subset \mathbb{P}^{n-1} .
$$

$M_{x}$ is smooth of dimension $n-1-d$ for general $x \in X$ by [Kol96, II.3.11]. Thus $M_{x}$ is a smooth complete intersection, hence irreducible if $n-1-d \geq 1$. 
Thus $M$ has a unique irreducible component $M^{0} \subset M$ such that the corresponding family $u^{0}: C_{M}^{0} \rightarrow X$ is dominant and has geometrically irreducible generic fiber. Thus, by Corollary $8 M^{0} \leftarrow C_{M}^{0} \stackrel{u^{0}}{\rightarrow} X$ satisfies the Lefschetz property.

Conversely, assume that $d \geq n-1$. If $d \geq n$ then there is no line through a general point; this is like example (3,1). The $d=n-1$ case is discussed in (3,4).

Remark 10. The proof applies to any smooth, Fano complete intersection of Fano index $\geq 3$. If the Fano index is 2, applying Remark 7 yields very high degree curves, but most likely conics work if the Fano index is 2 and cubics if the Fano index is 1.

As far as I know, Corollary 2 should hold in any characteristic. It holds for general hypersurfaces where the family of lines is smooth and has the expected dimension (cf. Kol96, V.4.3]).

The proof of Theorem 5 follows the outlines of [Kol13, Sec.5]. First we recall properties of open chains, then we pass to a subfamily that is topologically trivial. After studying which chains lift to étale covers, the proof is completed in Paragraphs 19]20. At the end we consider how to modify the statements and the proofs to work over arbitrary fields.

\section{Open chains.}

11 (Chains of varieties over $X$ ). Let $M \leftarrow C_{M} \stackrel{u}{\rightarrow} X$ be a family of schemes over $X$. A $C_{M}$-link is a morphism of a triple $u_{m}:\left(C_{m}, a, b\right) \rightarrow X$ where $m \in M$ and $a, b \in C_{m}$. A $C_{M}$-chain of length $r$ over $X$ consists of

(1) $C_{M}$-links $u_{i}:\left(C_{i}, a_{i}, b_{i}\right) \rightarrow X$ for $i=1, \ldots, r$ such that

(2) $u_{i}\left(b_{i}\right)=u_{i+1}\left(a_{i+1}\right)$ for $i=1, \ldots, r-1$.

We say that the chain starts at $u_{1}\left(a_{1}\right) \in X$ and ends at $u_{r}\left(b_{r}\right) \in X$ or that it connects $u_{1}\left(a_{1}\right)$ and $u_{r}\left(b_{r}\right)$.

A $C_{M}$-chain determines a reducible variety $\vee_{i=1}^{r} C_{i}$ obtained from the disjoint union of $C_{1}, \ldots, C_{r}$ by identifying $b_{i} \in C_{i}$ with $a_{i+1} \in C_{i+1}$ for $i=1, \ldots, r-1$. The morphisms $u_{i}$ then define a morphism $\vee_{i} u_{i}: \vee_{i} C_{i} \rightarrow X$. If the $C_{i}$ are connected then the image of $\vee_{i} u_{i}$ is a connected subscheme of $X$ which contains the starting and end points of the chain.

Starting with $M \leftarrow C_{M} \stackrel{u}{\rightarrow} X$ the set of all pairs $\left(C_{m}, a\right)$ (resp. triples $\left.\left(C_{m}, a, b\right)\right)$ is naturally given by

$$
C_{M} \leftarrow C_{M} \times_{M} C_{M} \stackrel{u \circ \pi_{2}}{\longrightarrow} X \quad \text { and } \quad C_{M} \times_{M} C_{M} \leftarrow C_{M} \times_{M} C_{M} \times_{M} C_{M} \stackrel{u \circ \pi_{3}}{\longrightarrow} X
$$

where the marked points are given by the diagonal maps

$$
\delta: C_{M} \rightarrow C_{M} \times_{M} C_{M} \quad \text { and } \quad \delta_{1}, \delta_{2}: C_{M} \times_{M} C_{M} \rightarrow C_{M} \times_{M} C_{M} \times_{M} C_{M} .
$$

Here $\pi_{i}$ denotes the $i$ th coordinate projection and $\delta_{i}$ maps the first $C_{M}$ identically to the $i$ th factor and the second $C_{M}$ diagonally to the other 2 factors. Thus all $C_{M}$-chains of length 1 are parametrized by

$$
C_{M} \times_{M} C_{M} \leftarrow C_{M} \times_{M} C_{M} \times_{M} C_{M} \stackrel{u \circ \pi_{3}}{\longrightarrow} X
$$

which we denote from now on by

$$
\text { Chain }\left(C_{M}, 1\right) \stackrel{p^{(1)}}{\longleftarrow} C_{M}^{(1)} \stackrel{u^{(1)}}{\longrightarrow} X .
$$

Out of this we get that all $C_{M}$-chains of length 2 are parametrized by

$$
\text { Chain }\left(C_{M}, 2\right):=\operatorname{Chain}\left(C_{M}, 1\right) \times_{X} \text { Chain }\left(C_{M}, 1\right)
$$


where the 2 maps Chain $\left(C_{M}, 1\right) \rightarrow X$ are given by $u^{(1)} \circ \delta_{2}$ on the first copy and $u^{(1)} \circ \delta_{1}$ on the second copy. Over this there is a universal family

$$
\text { Chain }\left(C_{M}, 2\right) \stackrel{p^{(r, 1)} \vee p^{(r, 2)}}{\longleftarrow} C_{M}^{(2,1)} \vee C_{M}^{(2,2)} \stackrel{u^{(r, 1)} \vee u^{(r, 2)}}{\longrightarrow} X
$$

where $C_{M}^{(r, i)}$ denotes the universal family of the $i$ th links of the $r$-chains.

By iterating this we get Chain $\left(C_{M}, r\right)$ parametrizing length $r$ chains

$$
\text { Chain }\left(C_{M}, r\right) \stackrel{\vee_{i} p^{(r, i)}}{\longleftarrow} \vee_{i=1}^{r} C_{M}^{(r, i)} \stackrel{\vee_{i} u^{(r)}}{\longrightarrow} X .
$$

If $C_{M} \rightarrow M$ is flat with irreducible fibers then $C_{M}^{(1)} \rightarrow$ Chain $\left(C_{M}, 1\right)$ is also flat with irreducible fibers. For a point $x \in X$ we have

$$
\left(u^{(1)}\right)^{-1}(x) \cong u^{-1}(x) \times_{M} C_{M} .
$$

Thus we conclude the following.

Claim 11.6. Assume that $M$ is irreducible and both maps $M \leftarrow C_{M} \rightarrow X$ are flat with irreducible fibers. Then:

a) Each Chain $\left(C_{M}, r\right)$ is irreducible.

b) The maps Chain $\left(C_{M}, r\right) \stackrel{p^{(r, i)}}{\longleftarrow} C_{M}^{(r, i)} \stackrel{u^{(r, i)}}{\longrightarrow} X$ are flat with irreducible fibers.

c) If $C_{M}^{0} \subset C_{M}$ is a dense open subset then Chain $\left(C_{M}^{0}, r\right)$ is a dense open subset of Chain $\left(C_{M}, r\right)$.

Definition 12. With the above notation, the starting and end points give morphisms

$$
\alpha, \beta: \text { Chain }\left(C_{M}, r\right) \rightarrow X .
$$

We say that $X$ is generically $C_{M}$-connected if

$$
\alpha \times \beta: \text { Chain }\left(C_{M}, r\right) \rightarrow X \times X
$$

is dominant for some $r$, that is, if two general points of $X$ can be connected by a $C_{M}$-chain of length $r$. (The equivalence of this definition with the one given in (3) 2) is proved in [Kol96, IV.4.13].)

If $u$ is open and $X$ is generically $C_{M}$-connected then $\alpha \times \beta$ is dominant for every $r \geq \operatorname{dim} X$ by $\underline{\text { Kol96, }}$ IV.4.13].

Note that if $X$ is generically $C_{M}$-connected, $M$ is irreducible and both maps $M \leftarrow C_{M} \rightarrow X$ are flat with irreducible fibers then, by (11,6.c), $X$ is also generically $C_{M}^{0}$-connected for every dense open subset $C_{M}^{0} \subset C_{M}$.

Now we choose an especially well behaved subset $C_{M}^{0} \subset C_{M}$.

Proposition 13. Let $X$ be a normal variety and $M \stackrel{p}{\leftarrow} C_{M} \stackrel{u}{\rightarrow} X$ a family of varieties over $X$ where $M$ is irreducible and both maps are flat with irreducible fibers. Let $\emptyset \neq X^{0} \subset X$ be an open subset. Then there is an open subset $\emptyset \neq C_{M}^{0} \subset$ $C_{M}$ with induced maps $p^{0}: C_{M}^{0} \rightarrow M$ and $u^{0}: C_{M}^{0} \rightarrow X$ such that

(1) $p^{0}$ is smooth with irreducible fibers,

(2) $p^{0}$ is a topologically locally trivial fiber bundle (over its image),

(3) the image of $u^{0}$ is contained in $X^{0}$ and

(4) $u^{0}$ has irreducible (hence connected) fibers. 
Proof. We first replace $C_{M}$ by the open subset $C_{M}^{1}=u^{-1}\left(X^{0}\right)$ and then by the open subset $C_{M}^{2} \subset C_{M}^{1}$ where $p$ is smooth.

By GM88, p.43], every map between algebraic varieties is a locally topologically trivial fiber bundle over a Zariski open subset. Thus by passing to an open subset $C_{M}^{0} \subset C_{M}^{2}$ we may assume that properties (1-3) hold. Since each fiber of $u$ is irreducible, the same holds for $u^{0}$.

The pointed fibers $\left(C_{m}^{0}, a\right)$ also form a topologically locally trivial fiber bundle $C_{M}^{0} \leftarrow C_{M}^{0} \times_{M} C_{M}^{0}$. Given a point $x \in X^{0}$, the set of all $\left(C_{m}^{0}, a\right)$ such that $u(a)=x$ form a topologically locally trivial fiber bundle over the connected base $\left(u^{0}\right)^{-1}(x)$. As we noted in (3.7),

$$
\operatorname{im}\left[\pi_{1}\left(C_{m}^{0}, a\right) \rightarrow \pi_{1}\left(X^{0}, x\right)\right]=\operatorname{im}\left[\pi_{1}\left(C_{m}^{1}, a\right) \rightarrow \pi_{1}\left(X^{0}, x\right)\right] .
$$

Thus we obtain the following.

Corollary 14. Notation and assumptions as in (13). Then, for every $m \in M$, $a \in C_{m}^{0}$ and $x:=u(a)$, the image of the induced map

$$
\Gamma\left(X^{0}, C, x\right):=\operatorname{im}\left[\pi_{1}\left(C_{m}^{0}, a\right) \stackrel{u_{*}^{0}}{\longrightarrow} \pi_{1}\left(X^{0}, x\right)\right] \subset \pi_{1}\left(X^{0}, x\right)
$$

depends only on $\left(X^{0}, x\right)$ and $C_{M}$ but not on $m \in M$ and $a \in C_{m}^{0}$.

\section{Topologically locally trivial families.}

15. We work with families $M \stackrel{p}{\leftarrow} C_{M} \stackrel{u}{\rightarrow} X$ such that $p$ has irreducible fibers and the following holds:

(15.1) For every $x \in X, m \in M$ and $a \in C_{m}$ satisfying $u_{m}(a)=x$, the image of the induced map

$$
\Gamma(X, C, x):=\operatorname{im}\left[\pi_{1}\left(C_{m}, a\right) \stackrel{u_{*}}{\longrightarrow} \pi_{1}(X, x)\right] \subset \pi_{1}(X, x)
$$

does not depend on $m \in M$ and $a \in C_{m}$.

An equivalent formulation is the following.

(15.2) Let $(\tilde{X}, \tilde{x}) \rightarrow(X, x)$ be any covering space such that

$$
u_{m}:\left(C_{m}, a\right) \rightarrow(X, x) \quad \text { lifts to } \tilde{u}_{m}:\left(C_{m}, a\right) \rightarrow(\tilde{X}, \tilde{x})
$$

for some $m \in M$ and $a \in C_{m}$. Then the lift exists for every $n \in M, b \in C_{n}$ for which $u_{n}(b)=x$.

Now fix a point $x \in X$. Corresponding to $\Gamma(X, C, x)$ there is an étale cover

$$
q_{X}:(\tilde{X}, \tilde{x}) \rightarrow(X, x) .
$$

We do not yet know that $\Gamma(X, C, x)$ has finite index, so $\tilde{X} \rightarrow X$ could have infinite degree. Thus $\tilde{X}$ is an analytic space for now.

Proposition 16. Notation and assumptions as in (15). Then every $C_{M}$-chain on $X$ starting at $x$ lifts to a $C_{M}$-chain on $\tilde{X}$ starting at $\tilde{x}$.

Proof. A $C_{M}$-chain is given by the data $u_{i}:\left(C_{i}, a_{i}, b_{i}\right) \rightarrow X$. Set $x_{1}:=x$. By the choice of $\Gamma\left(X, C, x_{1}\right)$,

$$
u_{1}:\left(C_{1}, a_{1}\right) \rightarrow\left(X, x_{1}\right) \quad \text { lifts to } \quad \tilde{u}_{1}:\left(C_{1}, a_{1}\right) \rightarrow\left(\tilde{X}, \tilde{x}_{1}\right) .
$$

If we let $\tilde{x}_{2}$ denote the image of $b_{1}$ then we can view the latter map as

$$
\tilde{u}_{1}:\left(C_{1}, b_{1}\right) \rightarrow\left(\tilde{X}, \tilde{x}_{2}\right) .
$$


We next apply (15) to

$$
u_{1}:\left(C_{1}, b_{1}\right) \rightarrow\left(X, x_{2}\right) \text { and } u_{2}:\left(C_{2}, a_{2}\right) \rightarrow\left(X, x_{2}\right)
$$

to see that if one of them lifts to $\left(\tilde{X}, \tilde{x}_{2}\right)$ then so does the other. This gives us

$$
\tilde{u}_{2}:\left(C_{2}, a_{2}\right) \rightarrow\left(\tilde{X}, \tilde{x}_{2}\right) .
$$

We can iterate the argument to lift the whole chain.

Corollary 17. Notation and assumptions as in (15). Assume in addition that $X$ is $C_{M}$-connected. Then $\Gamma(X, C, x) \subset \pi_{1}(X, x)$, as in 15.1), has finite index, thus $q_{X}:(\tilde{X}, \tilde{x}) \rightarrow(X, x)$ is an algebraic étale cover.

More precisely, the degree of $q_{X}$ is bounded by $N:=$ the number of irreducible components of the geometric generic fiber of $\alpha \times \beta$ : Chain $(X, \operatorname{dim} X) \rightarrow X \times X$.

Proof. Let Chain $\left(C_{M}, r, x\right) \subset$ Chain $\left(C_{M}, r\right)$ denote the subscheme parametrizing chains that start at $x$. Thus Chain $\left(C_{M}, r, x\right)$ is a fiber of $\alpha$ : Chain $\left(C_{M}, r\right) \rightarrow X$ and, for general $x \in X$, the number of irreducible components of the geometric generic fiber of $\beta$ : Chain $(X, r, x) \rightarrow X$ equals $N$.

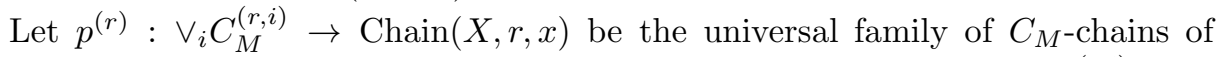
length $r$ with starting and end point sections $\alpha, \beta$ : Chain $(X, r, x) \rightarrow \vee_{i} C_{M}^{(r, i)}$.

Note that $u^{(r, 1)} \circ \alpha$ maps Chain $(X, r, x)$ to $\{x\}$ and, by (16), each fiber of $p^{(r)}$ lifts to a $\tilde{C}_{m}$-chain on $\tilde{X}$ starting at $\tilde{x}$. Thus

$$
\vee_{i} u^{(r, i)}: \vee_{i} C_{M}^{(r, i)} \rightarrow X \quad \text { lifts to } \vee_{i} \tilde{u}^{(r, i)}: \vee_{i} \tilde{C}_{M}^{(r, i)} \rightarrow \tilde{X} .
$$

In particular, the end point map

$$
u^{(r, r)} \circ \beta: \text { Chain }\left(C_{M}, r, x\right) \rightarrow X \quad \text { lifts to } \quad \tilde{u}^{(r, r)} \circ \tilde{\beta}: \operatorname{Chain}\left(C_{M}, r, x\right) \rightarrow \tilde{X} .
$$

Therefore

$$
\operatorname{im}\left[\beta_{*}: \pi_{1}\left(\operatorname{Chain}\left(C_{M}, r, x\right)\right) \rightarrow \pi_{1}(X)\right] \subset \Gamma(X, C, x) .
$$

By assumption (and Kol96, 4.13]) $\beta$ is dominant for $r \geq \operatorname{dim} X$. Therefore, by Kol95, 2.10], the index is bounded as

$$
\mid \pi_{1}(X): \operatorname{im}\left[\pi_{1}\left(\text { Chain }\left(C_{M}, r, x\right)\right) \rightarrow \pi_{1}(X)\right] \mid \leq N .
$$

18 (Proof of 4). The implication (4,1) $\Rightarrow$ (4,2) was already noted in (3,1-3).

It remains to show that (4.2) $\Rightarrow$ (4)3).

As we noted in (12), replacing $C_{M}$ with an open subset $\emptyset \neq C_{M}^{0} \subset C_{M}$ does not change the assumptions. Thus we may assume that the assumptions of (17) hold. This gives the bound $N:=$ the number of irreducible components of the geometric generic fiber of $\alpha \times \beta$ : Chain $(X, \operatorname{dim} X) \rightarrow X \times X$.

\section{Proof of Theorem 5 .}

Fix an open subset $X^{0} \subset X$ and use (13) to obtain $C_{M}^{0} \subset C_{M}$. Then pick a general point $x \in X^{0}$ and, as in Paragraph [15, construct

$$
q_{X}^{0}:\left(\tilde{X}^{0}, \tilde{x}\right) \rightarrow\left(X^{0}, x\right) .
$$

By Proposition 4, $q_{X}^{0}$ has finite degree, thus it extends (uniquely) to a normal, possibly ramified, finite cover

$$
q_{X}:(\tilde{X}, \tilde{x}) \rightarrow(X, x)
$$


If $q_{X}$ is also étale then $\tilde{X}^{0} \rightarrow X^{0}$ is the pull-back of the finite étale cover $\tilde{X} \rightarrow X$; this is what (5.4) asserts.

All that remains is to derive a contradiction if $q_{X}$ is ramified. Since $X$ is smooth, in this case there is a nonempty branch divisor $B \subset X$. First we show that most $C_{M}$-chains starting at $x$ lift to $\tilde{X}$. Then we use the branch divisor to show that most chains do not lift, thereby arriving at a contradiction.

19 (Lifting $C_{M}$-chains). A $C_{M^{-}}^{0}$-chain is given by the data $u_{i}^{0}:\left(C_{i}^{0}, a_{i}, b_{i}\right) \rightarrow X$. Here each $C_{i}^{0}$ is an open subset of the corresponding $C_{i}$ thus the $C_{M}^{0}$-chain naturally corresponds to a $C_{M}$-chain given by the data $u_{i}:\left(C_{i}, a_{i}, b_{i}\right) \rightarrow X$.

Since the $C_{i}$ are normal (even smooth) and $q_{X}: \tilde{X} \rightarrow X$ is finite, every lifting $\tilde{u}_{i}^{0}: C_{i}^{0} \rightarrow \tilde{X}$ of $u_{i}^{0}$ uniquely extends to $\tilde{u}_{i}: C_{i} \rightarrow \tilde{X}$. Thus if a $C_{M^{0}}^{0}$-chain lifts to $\tilde{X}^{0}$ then the corresponding $C_{M}$-chain also lifts to $\tilde{X}$.

20 (Non-liftable chains). Assume that the branch divisor $B_{X} \subset X$ of $q_{X}: \tilde{X} \rightarrow X$ is nonempty. Let $B_{X}^{*} \subset B_{X}$ be the open subset of smooth points. Since $u: C_{M} \rightarrow X$ is surjective and smooth, the preimage $B_{C}:=u^{-1}\left(B_{X}\right)$ is also nonempty and $u^{-1}\left(B_{X}^{*}\right)$ is smooth and nonempty. Let $B_{C}^{*} \subset u^{-1}\left(B_{X}^{*}\right)$ be the set of points where the restriction of $p$ to $B_{C}$ is smooth. Finally let $M^{*} \subset M$ be the open subset consisting of those points $m \in M$ such that $C_{m}$ meets $B_{C}^{*}$ in at least 1 point. Thus for $m \in M^{*}$ there is a map of the unit disc $\tau_{m}: \Delta \rightarrow C_{m}$ such that $u \circ \tau_{m}: \Delta \rightarrow X$ is transversal to $B$. Since $q_{X}$ branches along $B$, the sheets of $\tilde{X} \rightarrow X$ have nontrivial monodromy around $B$ and the pull-back to $\Delta$ still has nontrivial monodromy.

Set $d:=\operatorname{deg} \tilde{X} / X$. If $m \in M^{*}$ then the pull-back

$$
q_{m}: C_{m} \times_{X} \tilde{X} \rightarrow C_{m}
$$

is a degree $d$ cover that is étale outside $C_{m} \cap B_{C}^{*}$ and whose monodromy around $C_{m} \cap B_{C}^{*}$ is nontrivial. The cover need not be connected or normal, but, due to the monodromy, it can not be a union of $d$ trivial covers $C_{m} \cong C_{m}$. That is, if $a \in C_{m}$ is a general point and $\tilde{a}_{1}, \ldots, \tilde{a}_{d}$ its preimages in $C_{m} \times_{X} \tilde{X}$ then, for at least one $\tilde{a}_{i}$, the identity map $\left(C_{m}, a\right) \rightarrow\left(C_{m}, a\right)$ can not be lifted to $\left(C_{m}, a\right) \rightarrow\left(C_{m} \times_{X} \tilde{X}, \tilde{a}_{i}\right)$.

Thus if $y \in X$ is the image of $a$ and $\tilde{y}_{1}, \ldots, \tilde{y}_{d} \in \tilde{X}$ its preimages, then for at least one $\tilde{y}_{i}$, the map $u_{m}:\left(C_{m}, a\right) \rightarrow(X, y)$ can not be lifted to

$$
\tilde{u}_{(m, i)}:\left(C_{m}, a\right) \nrightarrow \rightarrow\left(\tilde{X}, \tilde{y}_{i}\right) \text {. }
$$

Consider now the dominant map $\tilde{\beta}_{r}:$ Chain $\left(C_{M}^{0}, r, x\right) \rightarrow \tilde{X}^{0}$ and let $X^{*} \subset X$ be a Zariski open subset such that $q_{X}^{-1}\left(X^{*}\right) \subset \operatorname{im} \tilde{\beta}_{r}$.

By choosing the above $u_{m}: C_{m} \rightarrow X$ generically, we may assume that there is a point $a_{r+1} \in C_{m}$ such that $y:=u_{m}\left(a_{r+1}\right) \in X^{*}$.

By the choice of $X^{*}$, for every $\tilde{y}_{i}^{*} \in q_{X}^{-1}(y)$ there is a $C_{M^{0}}^{0}$-chain of length $r$ whose lift to $\tilde{X}$ connects $\tilde{x}$ and $\tilde{y}_{i}^{*}$. We can add $u_{m}^{0}:\left(C_{m}^{0}, a_{r+1}, b_{r+1}\right) \rightarrow X$ as the last link of any of these chains. Thus we get $d$ different $C_{M}^{0}$-chains of length $r+1$ and, for at least one of them, its extension to a $C_{M}$-chain can not be lifted to $\tilde{X}$. This contradicts (19) and completes the proof of Theorem 5 .

\section{Other fields.}

Our results apply to varieties over an arbitrary field, with two modifications.

First, we have to use the algebraic fundamental group; which we still denote by $\pi_{1}$. Note that if $k$ is any field with algebraic closure $\bar{k}$ and $p: Y \rightarrow X$ is a morphism of geometrically irreducible $k$-varieties then the induced map $\pi_{1}(Y) \rightarrow$ 
$\pi_{1}(X)$ is surjective iff $\pi_{1}\left(Y \times_{k} \bar{k}\right) \rightarrow \pi_{1}\left(X \times_{k} \bar{k}\right)$ is surjective. Thus our questions are geometric in nature and the key point is to understand what happens over algebraically closed fields in positive characteristic.

The main difference is that even the classical Lefschetz theorem fails in the non-projective case. For instance, $\pi_{1}\left(\mathbb{A}^{1}\right) \rightarrow \pi_{1}\left(\mathbb{A}^{2}\right)$ is not surjective in positive characteristic. (An example is given by the cover $\left(z^{p}+z+x=0\right) \subset \mathbb{A}^{3}$ of the $x y$-plane which splits over any line $x=c$.) This is remedied with the following variant of Definition 1

Definition 21. Let $k$ be an algebraically closed field of positive characteristic and

$$
M \stackrel{p}{\leftarrow} C_{M} \stackrel{u}{\rightarrow} X .
$$

a family of schemes where $M$ is geometrically irreducible and $p$ is flat with irreducible fibers. We say that the family $(21,1)$ satisfies the Lefschetz property if the following holds.

For every Zariski open dense subset $X^{0} \subset X$ and every finite quotient $\pi_{1}\left(X^{0}\right) \rightarrow G$ there is a Zariski open dense subset $M_{G}^{0} \subset M$ such that, for every $m \in M_{G}^{0}$, the induced map

$$
u\left(X^{0}, G, m\right)_{*}: \pi_{1}\left(C_{m} \cap u^{-1}\left(X^{0}\right)\right) \rightarrow \pi_{1}\left(X^{0}\right) \rightarrow G \text { is surjective. }
$$

We say that (21,1) satisfies the weak Lefschetz property if there is a constant $N$ (independent of $X^{0}$ and of $G$ ) such that, for a suitable choice of $M_{G}^{0}$, the image of $u\left(X^{0}, G, m\right)_{*}$ has index at most $N$ in in $G$.

With this notion, the only question is what should replace the topologically trivial family used in (13). Topological triviality is used only through its consequence [15, 1). In our case we need that

$$
\Gamma(X, C, x):=\operatorname{im}\left[\pi_{1}\left(C_{m}, a\right) \rightarrow \pi_{1}(X, x) \rightarrow G\right] \subset G
$$

be independent of $m \in M$ and $a \in C_{m}$. This is an easy consequence of the semicontinuity property of the fundamental groups in fibers; see [Kol03, Prop.16] for a precise statement and proof.

The rest of the arguments go through with minor changes.

Acknowledgments. I thank J. Starr for useful comments. Partial financial support was provided by the NSF under grant number DMS-0968337.

\section{REFERENCES}

[BdS09] Indranil Biswas and João Pedro P. dos Santos, On the vector bundles over rationally connected varieties, C. R. Math. Acad. Sci. Paris 347 (2009), no. 19-20, 1173-1176. MR 2566998 (2011b:14087)

[BK08] V. Balaji and János Kollár, Holonomy groups of stable vector bundles, Publ. Res. Inst. Math. Sci. 44 (2008), no. 2, 183-211. MR 2426347 (2010c:14044)

[BK12] , Restrictions of stable bundles, Compact moduli spaces and vector bundles, Contemp. Math., vol. 564, Amer. Math. Soc., Providence, RI, 2012, pp. 177-184. MR 2894633

[CT00] Jean-Louis Colliot-Thélène, Rational connectedness and Galois covers of the projective line, Ann. of Math. (2) 151 (2000), no. 1, 359-373. MR 1745009 (2001b:14046)

[GM88] Mark Goresky and Robert MacPherson, Stratified Morse theory, Ergebnisse der Mathematik und ihrer Grenzgebiete (3), vol. 14, Springer-Verlag, Berlin, 1988. MR 932724 (90d:57039)

[Kol95] János Kollár, Shafarevich maps and automorphic forms, M. B. Porter Lectures, Princeton University Press, Princeton, NJ, 1995. MR 1341589 (96i:14016) 
[Kol96] - Rational curves on algebraic varieties, Ergebnisse der Mathematik und ihrer Grenzgebiete. 3. Folge., vol. 32, Springer-Verlag, Berlin, 1996.

[Kol00] _ Fundamental groups of rationally connected varieties, Michigan Math. J. 48 (2000), 359-368, Dedicated to William Fulton on the occasion of his 60th birthday.

[Kol03] L Rationally connected varieties and fundamental groups, Higher dimensional varieties and rational points (Budapest, 2001), Bolyai Soc. Math. Stud., vol. 12, Springer, Berlin, 2003, pp. 69-92.

[Kol13] - Neighborhoods of subvarieties in homogeneous spaces, ArXiv e-prints (2013).

[KS03] János Kollár and Endre Szabó, Rationally connected varieties over finite fields, Duke Math. J. 120 (2003), no. 2, 251-267.

Princeton University, Princeton NJ 08544-1000

kollar@math.princeton.edu 